\title{
Дифференциальные уравнения для восстановления производной безгистерезисной нелинейной вольт-амперной характеристики полупроводниковой структуры
}

\author{
(C) Н.Д. Кузьмичев, М.А. Васютин \\ Мордовский государственный университет им. Н.П. Огарева, \\ 430005 Саранск, Россия \\ E-mail: kuzmichevnd@yandex.ru
}

(Получена 5 апреля 2018 г. Принята к печати 25 июня 2018 г.)

\begin{abstract}
Изучена последовательность неоднородных дифференциальных уравнений для восстановления производной нелинейной вольт-амперной характеристики, правой частью которых является экспериментально полученная зависимость напряжения первой гармоники от постоянного тока. Такое напряжение возникает, например, на выходе нелинейной полупроводниковой структуры при одновременном пропускании через нее переменного и постоянного токов. На основе численных решений дифференциальных уравнений разработанная методика применена для восстановления производной вольт-амперной характеристики двух $p-n$-переходов, включенных антипараллельно.
\end{abstract}

DOI: 10.21883/FTP.2019.01.46997.8881

\section{1. Введение}

Ранее в работах [1-6] разработан метод модуляционного фурье-анализа (МФА). Данный метод применялся при изучении нелинейных свойств намагниченности и вольт-амперных характеристик (BАХ) высокотемпературных сверхпроводников. В оптике и ЭПР спектроскопии широко применяется модуляционная методика, разработанная на случай малых амплитуд модуляции, являющаяся частным случаем модуляционного фурьеанализа. Метод МФА используется в тех случаях, когда непосредственное измерение физической характеристики по каким либо причинам затруднено.

В статье [5] была получена производная ВАХ нелинейной полупроводниковой структуры с помощью экспериментально определенных высших гармоник Фурье напряжения отклика. В настоящей работе производная BAX нелинейной полупроводниковой структуры впервые восстановлена путем численного решения неоднородных дифференциальных уравнений 2-го и 4-го порядков, правой частью которых является зависимость напряжения первой гармоники от постоянного тока.

\section{2. Дифференциальные уравнения для восстановления вольт-амперной характеристики}

При исследовании безгистерезисной ВАХ нелинейной структуры, например, двух антипараллельно включенных $p-n$-переходов, через структуру одновременно пропускается постоянный Іи переменный ток амплитудой $i$, т.е. полный ток равен: $J=I+i \cos (\omega \tau)$. Здесь $\omega-$ циклическая частота переменного тока, $\tau-$ время. В силу нелинейности ВАХ $V(J)$ на выходе структуры возникает негармонический периодический сигнал, в составе которого имеются первая и высшие гармоники [1-6]:

$$
\begin{aligned}
V(I+i \cos t) & =\sum_{n=0}^{\infty} \frac{i^{n} \cos ^{n} t}{n !} V^{(n)}(I) \\
& =\frac{A_{0}}{2}+\sum_{m=l}^{\infty} A_{m} \cos (m t),
\end{aligned}
$$

где $t=\omega \tau$ и

$$
A_{m}(I, i)=2 \sum_{n=0}^{\infty} \frac{1}{n !(n+m) !}\left(\frac{i}{2}\right)^{2 n+m} V^{(2 n+m)}(I) .
$$

Здесь функции $V^{(k)}(I)$ являются производными $k$-го порядка от BAX $V(I)$.

Амплитуда напряжения первой гармоники $A_{1}(I, i)$, согласно формуле (2), равна

$$
A_{1}(I, i)=2 \sum_{n=0}^{\infty} \frac{1}{n !(n+1) !}\left(\frac{i}{2}\right)^{2 n+1} V^{(2 n+1)}(I) .
$$

В формуле (3) оставим три первых члена разложения:

$$
A_{1}(I, i) \approx i V^{(1)}(I)+\frac{i^{3}}{8} V^{(3)}(I)+\frac{i^{5}}{192} V^{(5)}(I) .
$$

В случае очень малых амплитуд модуляции $i$ зависимость $A_{1}(I, i) / i$ с большой точностью совпадает с производной ВАХ равной $d V(I) / d I=V^{(1)}(I)$. Восстановление ВАХ осуществляется простым интегрированием зависимости $A_{1}(I) / i$. В случае увеличения амплитуды модуляции существенным оказывается второй член разложения (4) и для восстановления производной ВАХ необходимо решать дифференциальное уравнение:

$$
\frac{d^{3} V}{d I^{3}}+\frac{8}{i^{2}} \frac{d V}{d I}=\frac{8}{i^{3}} A_{1}(I, i) .
$$

Данное уравнение сводится к уравнению 2-го порядка путем замены $Z=V^{(1)}$. 
При дальнейшем увеличении $i$ необходимо учитывать третий член разложения и так далее. Каждый раз это приводит к увеличению порядка дифференциального уравнения на два. Для определения порядка уравнения можно воспользоваться следующей оценкой. В работе [5] для экспериментального восстановления производной BAX применялась формула $[3,4]$

$$
\frac{d V}{d I}=\frac{1}{i} \sum_{m=1}^{\infty}(-1)^{m-1}(2 m-1) A_{2 m-1}(I, i) .
$$

Учитывая, что в выражении (2) разложение амплитуды $m$-й гармоники начинается с $m$-й производной (наибольший вклад дает первый член ряда), отношение первого ко второму члену уравнения (5) можно заменить отношением членов ряда (6) для амплитуд 3-й и 1-й гармоник. Значения амплитуд определяются экспериментально. Таким образом, если $3\left|A_{3} / A_{1}\right| \sim 1$, а $5\left|A_{5} / A_{1}\right| \ll 1$, необходимо решать уравнение (5). Если величина $3\left|A_{3} / A_{1}\right| \ll 1$, производная ВАХ (дифференциальное сопротивление) определяется простым выражением: $d V / d I \approx A_{1}(I, i) / i$.

Другая более грубая оценка основана на формулах (2) и (4). Для 3-й и 5-й гармоник в (2) оставим первые члены: $A_{3} \approx \frac{i^{3}}{24} V^{(3)}(I)$ и $A_{5} \approx \frac{i^{5}}{1920} V^{(5)}(I)$. Беря отношения членов в выражении (4), получим следующие оценки. Если $3\left|A_{3} / A_{1}\right| \sim 1$, а $10\left|A_{5} / A_{1}\right| \ll 1$, решается уравнение (5). При $10\left|A_{5} / A_{1}\right| \sim 1$ необходимо решать дифференциальное уравнение:

$$
\frac{d^{5} V}{d I^{5}}+\frac{24}{i^{2}} \frac{d^{3} V}{d I^{3}}+\frac{192}{i^{4}} \frac{d V}{d I}=\frac{192}{I^{5}} A_{1}(I, i)
$$

Данное уравнение сводится к уравнению 4-го порядка путем замены $Z=V^{(1)}$.

Правая часть уравнений (5) и (7) $A_{1}(I, i)$ определяется экспериментально и задается численно. Для численного решения уравнений (5) и (7) необходимо задавать начальные условия (задача Коши). В уравнении (5) задаются значения производных $V^{(1)}\left(I_{0}\right)$ и $V^{(2)}\left(I_{0}\right)$.

Начальные условия определяются приближенно из теоретических соображений либо из эксперимента по измерению величины соответствующей гармоники напряжения. Для этого необходимо воспользоваться выражением (2). Оставляя первые члены разложения для $V^{(1)}\left(I_{0}\right)$ и $V^{(2)}\left(I_{0}\right)$, получим оценки:

$$
V^{(1)}\left(I_{0}\right) \approx \frac{1}{i} A_{1}\left(I_{0}, i\right) \text { и } V^{(2)}\left(I_{0}\right) \approx \frac{4}{i^{2}} A_{2}\left(I_{0}, i\right) .
$$

Значения $A_{1}$ и $A_{2}$ определяются экспериментально. Для более точной экспериментальной оценки начальных условий необходимо учитывать следующие члены ряда (2).

\section{3. Определение производной вольт-амперной полупроводниковой структуры, состоящей из 2 встречно включенных $p-n$-переходов}

Для демонстрации развитой методики восстановления BAX в качестве нелинейной структуры были взяты два встречно включенных полупроводниковых $p-n$-переходов. Более подробно экспериментальная методика и установка для измерений рассмотрены в работе [5].

Через вышеуказанную структуру пропускались переменный и постоянный токи $J=I+i \cos (\omega \tau)$. Амплитуда переменного тока изменялась в пределах $0<i \leq 2$ мА. Постоянный ток изменялся в пределах $0 \leq I \leq 10$ мА. Экспериментальная ВАХ используемой структуры и ее аналитическая зависимость приведены в работе [5]:

$$
V(I)=V_{0} \ln \left[\frac{I}{J_{0}}+\sqrt{\left(\frac{I}{J_{0}}\right)^{2}+1} .\right.
$$

Здесь $V_{0}=36.3 \mathrm{мB}$ и $J_{0}=0.9 \mathrm{мA}$.

Результаты выполненных измерений амплитуд напряжений первой гармоники, поделенных на амплитуду модуляции, равную $i$, приведены на рис. 1-3. Кроме того, на этих же рисунках приведены результаты восстановления производной нелинейной ВАХ изучаемой полупроводниковой структуры.

Пусть $i=1$ мА. Для этого случая $10\left|A_{5} / A_{1}\right| \approx 0.03 \ll 1$. В начальном грубом приближении дифференциальное сопротивление $V^{(1)}(I)$ структуры оценивалось по формуле (8). Следующим этапом процедуры восстановления является решение уравнения (5). Данное уравнение приводилось к системе уравнений 2-го порядка и

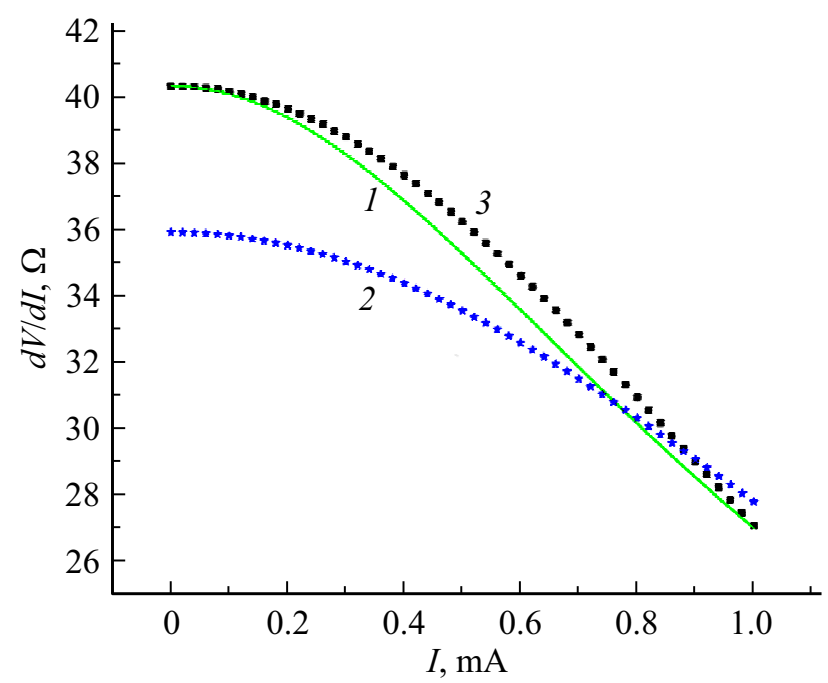

Рис. 1. Дифференциальное сопротивление $d V / d I$ (сплошная кривая 1), отношение амплитуды первой гармоники к амплитуде модуляции $A_{1}(I) / i$ (звездочки 2 ) и восстановленная $d V / d I$ на основе уравнения (5) (квадраты 3 ). Амплитуда модуляции $i=1$ мА и $I_{0}=0$. 


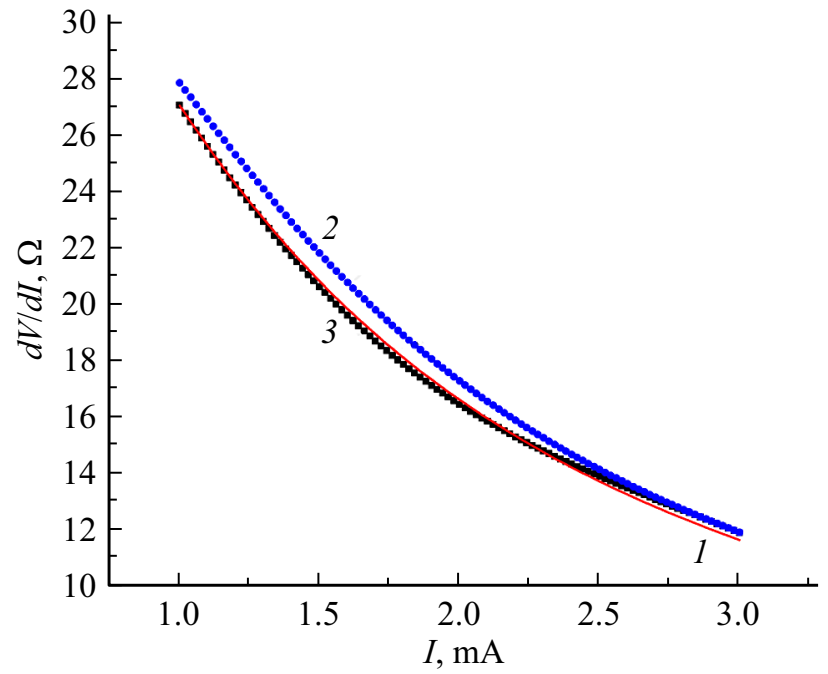

Рис. 2. Дифференциальное сопротивление $d V / d I$ (сплошная кривая 1), отношение амплитуды первой гармоники к амплитуде модуляции $A_{1} / i$ (кружки 2) и восстановленная $d V / d I$ на основе уравнения (5) (квадраты 3). Амплитуда модуляции $i=1$ мА и $I_{0}=1 \mathrm{мA}$.

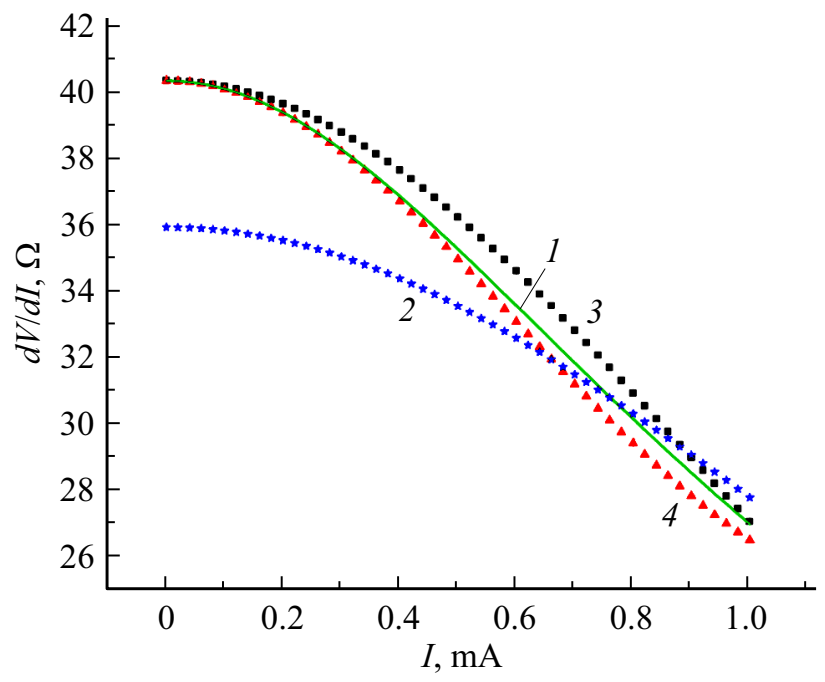

Рис. 3. Дифференциальное сопротивление $d V / d I$ (сплошная кривая 1), отношение амплитуды первой гармоники к амплитуде модуляции $A_{1} / i$ (звездочки 2 ), восстановленная $d V / d I$ на основе уравнения (5) (квадраты 3) и восстановленная $d V / d I$ на основе уравнения (7) (треугольники 4). Амплитуда модуляции $i=1 \mathrm{мA}$ и $I_{0}=0$.

решалось численно методом Рунге-Кутты 4-го порядка точности в системе MathCad. За начальные условия при $I=I_{0}=0$ принимались следующие значения: $V^{(1)}(0) \approx A_{1}(0) / i \approx 35.9$ Ом и и $V^{(2)}(0)=4 A_{2}(0) / i^{2}=0$, так как в нашем случае при $I=0$ четные гармоники отсутствовали. Для улучшения согласия значение $V^{(1)}(0)$ изменялось. В результате было получено значение $V^{(1)}(0) \approx 40.3$ Ом, что хорошо согласуется с формулой (9). Из (9) следует величина $V^{(1)}(0)=V_{0} / J_{0}$. Резуль- таты моделирования производной ВАХ приведены на рис. 1. Из рисунка видно, что грубая оценка дифференциального сопротивления по формуле $d V / d I=A_{1}(I) / i$ (кривая 2) сильно отличается от производной $\mathrm{BAX}$ (кривая 1). Производная ВАХ, определенная из уравнения (5) (кривая 3), хорошо согласуется с кривой 1 . В области $I>1 \mathrm{MA}$ расхождение кривых 1 и 3 увеличивается, что обусловлено погрешностью численного метода решения дифференциального уравнения. Для улучшения согласия необходимо сместить начальную точку $I_{0}$.

Для определения производной $\mathrm{BAX}$ в области $I>1 \mathrm{мA} \mathrm{начальные} \mathrm{условия} \mathrm{берем} \mathrm{при} I_{0}=1 \mathrm{мA}$. В этом случае $V^{(1)}(1 \mathrm{MA}) \approx 27$ Ом и $V^{(2)}(1 \mathrm{MA}) \approx-15 \mathrm{Oм} / \mathrm{MA}^{2}$. Результаты расчета для $I>1 \mathrm{мA}$ приведены на рис. 2. В области $1 \mathrm{MA}<I<1 \mathrm{MA}+i$ наблюдается согласие кривых 1 и 3.

Для лучшего согласия экспериментальной и восстановленной производных ВАХ, как было отмечено в разд. 2, необходимо решать уравнение (7). Для демонстрации этого случая было выполнено математическое моделирование при амплитуде модуляции $i=1 \mathrm{мA} \mathrm{и}$ $I_{0}=0$. Уравнение (7) сводилось к системе уравнений 4-го порядка с начальными условиями:

$$
\begin{gathered}
V^{(1)}(0)=40.3 \text { Ом }, \quad V^{(2)}(0)=V^{(4)}(0)=0, \\
V^{(3)}(0)=49.8 \mathrm{OM} / \mathrm{MA}^{2} .
\end{gathered}
$$

Вышеуказанная система уравнений решалась численно методом Рунге-Кутты 4-го порядка точности в системе MathCad. Результаты численных расчетов приведены на рис. 3. Из рисунка видно, что наилучшее согласие с экспериментальной производной ВАХ (сплошная кривая 1) наблюдается у кривой 4, которая получена путем решения дифференциального уравнения (7).

\section{4. Заключение}

В работе впервые показано, что на основании ряда (3) для амплитуды первой гармоники напряжения можно построить последовательность неоднородных дифференциальных уравнений, при численном решении которых удается восстанавливать производную ВАХ (дифференциальное сопротивление) с различной степенью точности. Правой частью вышеуказанных уравнений является экспериментально полученная зависимость первой гармоники напряжения от величины постоянного тока при фиксированной амплитуде модуляции. Применение развитой методики восстановления ВАХ к нелинейной полупроводниковой структуре показало хорошую точность, быстро увеличивающуюся с ростом порядка уравнения. Данную методику можно применять для исследования сверхпроводников, магнитных и сегнетоэлектрических материалов. 


\section{Список литературы}

[1] Н.Д. Кузьмичев. Письма ЖТФ, 17 (7), 56 (1991).

[2] Н.Д. Кузьмичев. ЖТФ, 64, (12), 63 (1994).

[3] Н.Д. Кузьмичев. Письма ЖТФ, 20 (22), 39 (1994).

[4] Н.Д. Кузьмичев. ЖТФ, 37 (7), 124 (1997).

[5] Н.Д. Кузьмичев, М.А. Васютин, Д.А. Шилкин. ФТП, 50 (6), 830 (2016).

[6] Н.Д. Кузьмичев. Журн. средневолжского мат. о-ва (ЖСВМО), $13(2), 70$ (2011).

Редактор Г.А. Оганесян

\section{Differential equations \\ for the reconstruction of the derived \\ non-hissterase nonlinear \\ $I-V$ characteristics of the semiconductor structure}

N.D. Kuzmichev, M.A. Vasyutin

Ogarev Mordovia State University, 430005 Saransk, Russia

Abstract In the paper, a sequence of inhomogeneous differential equations for the reconstruction of the derivative of the nonlinear $I-V$ characteristic is studied, the right-hand side of which is the experimentally obtained dependence of the voltage of the first harmonic on the direct current. Such voltage arises, for example, at the output of a nonlinear semiconductor structure wile simultaneously passing through it an alternating and direct current. On the basis of numerical solutions of differential equations, the developed technique is used to reconstruct the derivative of the $I-V$ characteristic of two $p-n$-junctions that are included antiparallel. 